\title{
MAGNETIC PROPERTIES OF YBCO-PST COMPOSITES
}

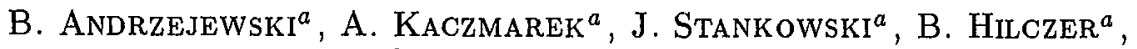 \\ J. Marfaing ${ }^{b}$, S. RéGnieR ${ }^{b}$ and C. Caranoni ${ }^{b}$ \\ ${ }^{a}$ Institute of Molecular Physics, Polish Academy of Sciences \\ Smoluchowskiego 17, 60-179 Poznań, Poland \\ ${ }^{b}$ Laboratoire: Matériaux, Organisation et Propriétés (MATOP) associé au CNRS \\ Case 151, Faculté des Sciences et Techniques, 13397 Marseille Cedex 20, France
}

(Received September 11, 2000; in final form November 11, 2000)

The magnetic properties of granular superconductor-insulator composites were investigated. The composites were random composition of the $\mathrm{YBa}_{2} \mathrm{Cu}_{3} \mathrm{O}_{7-\delta}$ (YBCO) high-temperature superconductor and $\mathrm{Pb}\left(\mathrm{Sc}_{0.5} \mathrm{Ta}_{0.5}\right) \mathrm{O}_{3}$ (PST) ferroelectric insulator. The composites exhibited lower superconducting volume ratio than the YBCO content. For samples with YBCO content low enough no global (the whole sample) and local (separated grains) superconductivity was observed. The observed decay of the superconductivity, lowering of the critical temperature, and the increase in number of paramagnetic centres as PST content increases are explained assuming reaction among YBCO and PST phases and possible formation of an additional ones, which did not exist in the initial powders.

PACS numbers: 74.25. Ha, 74.80.Bj, 76.30.Fc

\section{Introduction}

Poor mechanical, thermal and electromagnetic properties of high-temperature superconductors have to be improved for various practical applications. The large-scale applications such as power generation and storage, transportation, and large magnets need bulk high-temperature superconductors of high strength to withstand working stresses induced by high current and magnetic fields. On the other hand, for small scale and microelectronics applications one needs high-temperature superconductors of adequate electromagnetic properties and high thermal and electric conductivity.

The effective way for an improvement of high-temperature superconductors is formation of the superconducting composites. Normal state and superconducting properties of the composite depend both on the relative content and properties of 
the superconducting and nonsuperconducting phases in the composite and therefore can be easily changed.

Unfortunately, improvement of the mechanical properties of the composite often goes hand in hand with the decay of its superconductivity. The decreased critical temperatures and critical current densities were reported for most of the composites.

The insufficient grain-to-grain coupling was responsible for the loss of extrinsic superconducting properties, while the decay of the intrinsic ones was related to the ion interdiffusion. For example Regnier and Marfaing [1] reported a formation of the $\mathrm{Mn}$ oxides and other secondary phases during sintering of $\mathrm{YBCO} / \mathrm{Mn}$ composites. They concluded that $\mathrm{Mn}$ has a great facility to combine either with $\mathrm{Y}$ or Ba elements/oxides and to react with $\mathrm{Cu}$.

In this article we present the synthesis and investigations of the magnetic properties of the YBCO/PST superconductor/ferroelectric insulator composites of various YBCO to PST content. The observed effect such as: decay of the amount of the superconducting phase, lowering of the critical temperature and the increase in number of paramagnetic centres as PST content increases are explained assuming reaction between PST and YBCO phases.

\section{Sample preparation and experimental}

The composites were formed by mixing the YBCO and PST powders. Different concentrations were obtained by varying the YBCO weight ratio $\Phi_{\mathrm{W}}$ in the powder mixture: $\Phi_{\mathrm{W}}=0.2,0.25,0.5,0.75,1.0$.

Then the mixture was pressed at room temperature. The thermal cycle consisted in heating (in argon atmosphere) up to $900^{\circ} \mathrm{C}$ during $50 \mathrm{~min}$ at a heating rate of $18^{\circ} \mathrm{C} / \mathrm{min}$, then the sintering temperature in oxygen atmosphere was decreased at a rate of $7^{\circ} \mathrm{C} / \mathrm{min}$ with a step at $450^{\circ} \mathrm{C}$ during two hours. The samples were cooled down to $200^{\circ} \mathrm{C}$ at a rate of $1.5^{\circ} \mathrm{C} / \mathrm{min}$ and air quenched.

Systematically, various characterisations were carried out on the samples:

i) scanning electron microscopy study with energy dispersion spectroscopy analysis and X-ray diffraction measurements were performed at MATOP;

ii) AC/DC susceptibility measurements and EPR study were performed at IFM PAN.

X-ray diffraction patterns were performed on D 5000 Siemens equipment with $\mathrm{Ni}$ filtered lambda $\mathrm{Cu} K_{\alpha}$ radiation. Identification of the diffractograms gives evidence that the main peaks of the two oxides are present, with relative intensities corresponding to the pure phases. The initial nominal composition of YBCO was identified as 6.8 in oxygen concentration. After sintering, it appears that the PST reflections mostly correspond to the disordered phase [2]. However, the traces of an additional phase corresponding to a modified pyrochlore phase appeared after the sintering. It was identified as $\mathrm{Pb}_{14} \mathrm{Ta}_{10} \mathrm{O}_{39}$ by JCPDS File [3] and did not exist in the initial PST powder.

The magnetometric measurements were performed using AC susceptometer/DC magnetometer MagLab 2000 System. To compare the data obtained, all 
the samples were cylindrical in shape. The AC and DC magnetic fields were applied parallel to the symmetry axes of the cylinders. The aspect ratios $\gamma$ (ratio of the length to the diameter of the cylinder) and demagnetising factors $N$ of all samples were similar: $0.6<N<0.75$.

The study of the EPR were performed by means of standard X-band ( $9.4 \mathrm{GHz})$ spectrometer using continuous wave mode.

\section{Results and discussion}

Figure 1 illustrates dependence of magnetisation on magnetic field $M(H)$ for the YBCO/PST composites with YBCO nominal weight ratio $\Phi_{\mathrm{W}}=1.0,0.75,0.5$, $0.25,0.2$. The measurements were performed at the temperature $T=20 \mathrm{~K}$, which was well below the transition temperatures of the composites, except the nonsuperconducting composite of YBCO nominal content $\Phi_{\mathrm{W}}=0.2$. The $\Phi_{\mathrm{W}}=0.75$ composite exhibits stronger diamagnetism than pure $\mathrm{YBCO}$ and the highest critical current [4].

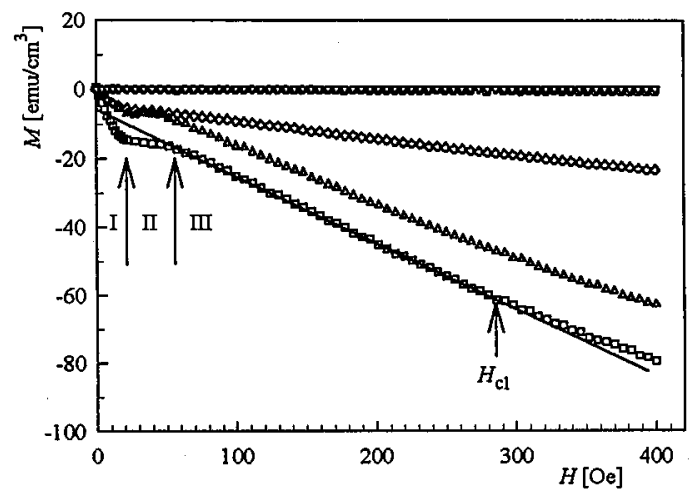

Fig. 1. Magnetisation $M(H)$ of the composites with respect to the YBCO nominal weight ratio $\mathrm{YBCO} \Phi_{\mathrm{W}}: \Delta-1.0, \square-0.75, \diamond-0.5, \bigcirc-0.25, \nabla-0.2$. The three limits: Meissner - I, kink — II, and linear - III are indicated.

The three limits occur in the $M(H)$ dependence for YBCO/PST composites when superconducting:

i) Nearly Meissner dependence is exhibited in the low field limit. The field where the magnetisation starts to deviate from Meissner-like behaviour ranges from 0 Oe for YBCO/PST composite with $\Phi_{\mathrm{W}}=0.25$ to 28 Oe for the composite characterised by $\Phi_{\mathrm{W}}=1.0$. The intergrain currents in this limit screen the total volume of the sample.

ii) The kink in $M(H)$ appears when the magnetic flux starts to slip between the superconducting grains (Fig. 1).

iii) Linear dependence in $M(H)$ is exhibited for fields lower than the critical field of the grains $H_{\mathrm{c} 1} \approx 280$ Oe but sufficiently high to almost suppress the 
intergranular supercurrents. Only the superconducting grains/phases give the contribution to the total magnetisation of the superconductor.

According to Yaron et al. [5] the relationship between magnetisation $M$ and the volume ratio $\Phi_{\mathrm{V}}$ of the superconducting phase (the volume of the superconducting phase to the total volume of the sample) in the third limit is as follows:

$$
M=-\alpha H,
$$

where

$$
\alpha=\frac{1}{4 \pi} \frac{\Phi_{\mathrm{V}}}{1-\Phi_{\mathrm{V}} N-\left(1-\Phi_{\mathrm{V}}\right) n},
$$

$N$ and $n$ denote the demagnetising coefficients of the sample and of the superconducting phase, respectively.

For YBCO/PST composites linear magnetisation in the third limit does not vanish when approximating to zero magnetic field and the Yaron model (1) should be modified

$$
M=-\alpha\left(\Phi_{\mathrm{V}}\right) H+M_{0},
$$

where the $\alpha$ coefficient has the same form as in (1) and $M_{0}$ is the value of magnetisation for zero magnetic field.

The non-vanishing term of the magnetisation $M_{0}$ is probably due to the existence in the third regime of the non-zero intergranular supercurrents contributing to the total magnetisation of the sample.

Regarding the modification in Eq. (2), the volume ratio of the superconducting phase can be described by the equation

$$
\Phi_{\mathrm{V}}=\frac{(1-n)\left(M-M_{0}\right)}{\left(M-M_{0}\right)(N-n)-H / 4 \pi} .
$$

To evaluate the volume ratio $\Phi_{\mathrm{V}}$ on the basis of Eq. (3) the spherical symmetry of the superconducting grains and the corresponding demagnetising factor $n=1 / 3$ are assumed. Figure 2 illustrates the volume ratio of the superconducting phase determined using Eq. (3) with respect to the YBCO nominal weight ratio $\Phi_{\mathrm{W}}$ in YBCO-PST composite.

None of the samples exhibited the superconducting volume ratio $\Phi_{\mathrm{V}}$ higher than 0.5 . The low $\Phi_{\mathrm{V}}$ volume is caused by high sample's porosity, by the London flux penetration into the superconducting grains taking place on defects even below $H_{\mathrm{c} 1}$ and by the gradual decay of the superconductivity as the PST content increases.

For samples of low YBCO weight ratio $\Phi_{\mathrm{W}}<0.25$ no global (the whole sample) and local (separated grains) superconductivity was observed.

The magnetic measurements performed in normal state revealed existence of the paramagnetic moment in YBCO-PST composites. If the origin of the magnetic moment is only due to the YBCO paramagnetic impurities (mainly "green phase" $\mathrm{Y}_{2} \mathrm{BaCuO}_{5}[6]$ ) the magnetic susceptibility per gram of YBCO should be constant.

The normalised susceptibility and integral EPR signal intensity dependence on composition of the YBCO-PST composites are shown in Fig. 3 [7].

The value of the normalised susceptibility is more or less constant for YBCO ratio $\Phi_{\mathrm{W}}$ over 0.5 and equal to $\chi \approx 10^{-5} \mathrm{emu} / \mathrm{g}_{\mathrm{YBCO}}$. For higher fractions of PST 


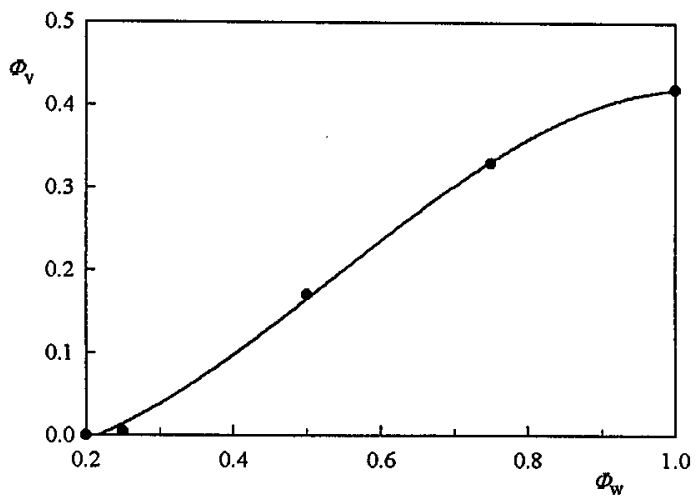

Fig. 2. Dependence of the superconducting fraction $\Phi_{\mathrm{V}}$ on the YBCO nominal weight ratio YBCO $\Phi_{\mathrm{W}}$. Solid line is the guide for eyes, only.

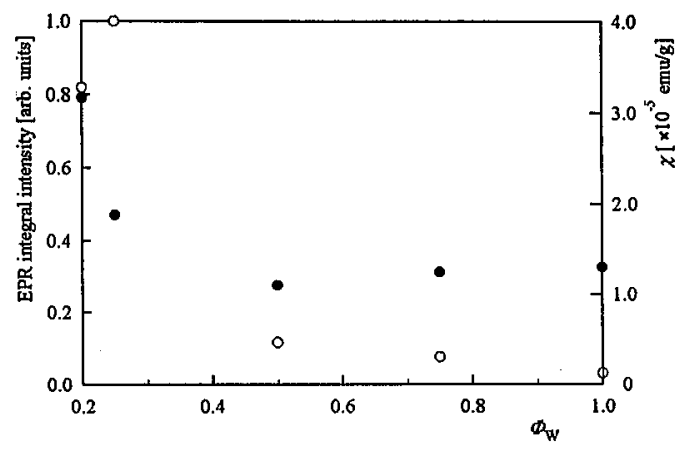

Fig. 3. EPR signal integral intensity ( 0 ) and magnetic susceptibility of the composites (•). Data are taken from Ref. [7].

in the composite the paramagnetism rapidly increases and reaches the value of $\chi \approx 3 \times 10^{-5} \mathrm{emu} / \mathrm{gYBCO}$ for the nonsuperconducting sample with YBCO ratio $\Phi_{\mathrm{W}}=0.2$.

Alongside with the increase in the paramagnetic moment increases the EPR integral signal intensity.

Figure 4 shows EPR spectra of the composites recorded at $100 \mathrm{~K}$. Both $g$-factor and EPR line width are constant and unaffected by the composition of the composites when $\Phi_{\mathrm{W}}>0.5$.

On decreasing the YBCO content the $g$-factor increases from $g \approx 2.06$ for YBCO-PST samples with $\Phi_{\mathrm{W}}$ below 0.5 to $g \approx 2.13$ for the nonsuperconducting one characterised by $\Phi_{\mathrm{W}}=0.2$.

In general, the strong anisotropic signal observed for $\Phi_{\mathrm{W}}=1.0$ characterised by axial symmetry $\left(g_{\|}>g_{\perp}\right)$ gives the evidence for elongated octahedral or square co-ordination. For $\Phi_{\mathrm{W}}=0.2$, the measured $g$-factor equal to $g \approx 2.13$ is the average of $g_{\|}$and $g_{\perp}$. In this case the co-ordination is the superposition of the two orbital states $\left|x^{2}-y^{2}\right\rangle$ and $\left|3 z^{2}-r^{2}\right\rangle$ as discussed in [8]. 


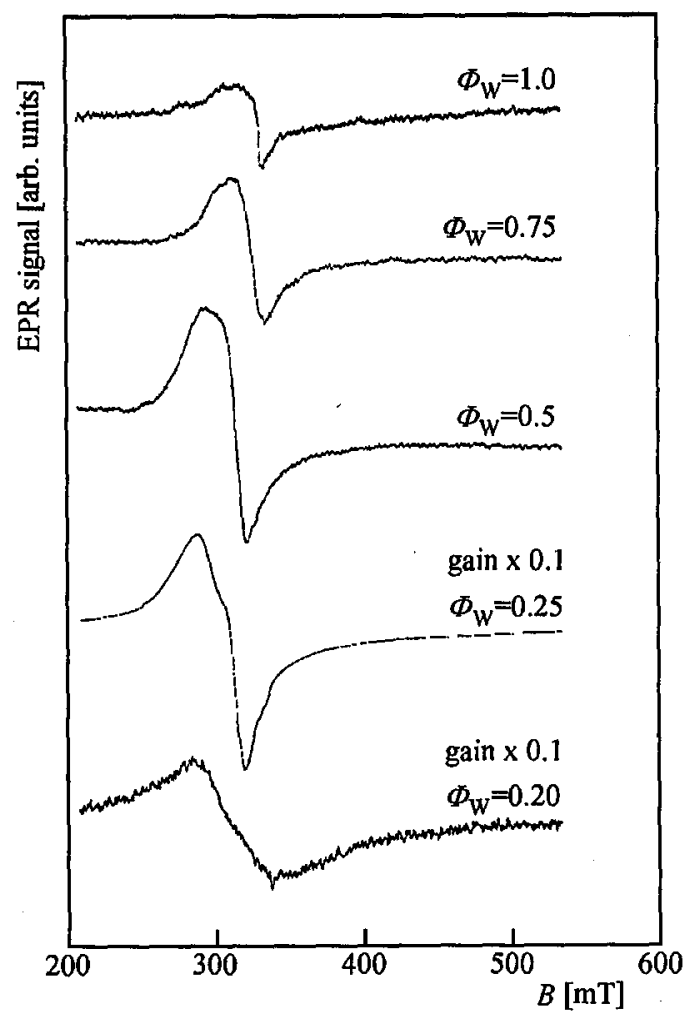

Fig. 4. EPR spectra of the composites recorded at $100 \mathrm{~K}$.

The changes in the $g$-factor and the increase in the EPR line width and intensity are caused by the progress of the reaction between YBCO and PST when $\Phi_{\mathrm{W}}<0.5$. Possible products of the reaction can be identified on the basis of the EPR spectra analysis.

The EPR spectrum of the sample $\Phi_{\mathrm{W}}=1.0$ is of axial or rhombic symmetry very close to $\mathrm{Y}_{2} \mathrm{BaCuO}_{5}$ "green phase" spectrum of rhombic symmetry characterised by $g_{z}=2.24, g_{y}=2.07, g_{x}=2.06$ [9]. "Green phase" usually appears during sintering of the pure YBCO and was actually found in the X-ray spectra of the composites $[6,10]$. The EPR spectrum of YBCO-PST sample $\Phi_{\mathrm{W}}=0.75$ is similar to the previous one. This is consistent with $\mathrm{X}$-ray investigations that revealed no modification for YBCO content $\Phi_{\mathrm{W}}>0.75$.

The spectrum changes for lower YBCO content. For the composite of $\Phi_{\mathrm{W}}=0.50$ the spectrum is a superposition of wide and narrow EPR lines characterised by axial symmetry. The broad one with peak-to-peak line width $\Delta B_{\mathrm{pp}}=$ $27 \mathrm{mT}$ may be due to presence of $\mathrm{BaCuO}_{2}$. The two well resolved lines are present for the composite of $\Phi_{\mathrm{W}}=0.25$. The spectrum of the nonsuperconducting YBCO-PST sample $\Phi_{\mathrm{W}}=0.20$ consists of the only one nearly symmetrical, broad line with the width $\Delta B_{\mathrm{pp}} \approx 50 \mathrm{mT}$ and centred at $g \approx 2.13$. 
The formation of the minor phases is probably caused by the ion diffusion. This is in line with the results of X-ray studies where Ba-rich regions inside PST phase were found [6]. Moreover, Svensson reported that the amount of $\mathrm{ScBaCuO}_{3.5}$ and $\mathrm{BaCuO}_{2}$ and $\mathrm{CuO}$ phases increases with the rise of Sc content [11]. On the other hand, the large magnetic moment equivalent to 2.7 spin per $\mathrm{Sc}$ atom and EPR spectra for $\mathrm{Y}_{1-x} \mathrm{Sc}_{x} \mathrm{Ba}_{2} \mathrm{Cu}_{3} \mathrm{O}_{7}$ compounds $(x=0.25,0.5)$ characterised by $g$ around 2 were observed by Zuo et al. [12].

The critical temperature determined using $\mathrm{AC}$ susceptibility method decreases as YBCO content decreases - see Fig. 5. The little disparity between the present results and earlier microwave measurements of $T_{\mathrm{c}}[13]$ may be due to sample ageing. On the other hand, AC method is sensitive to the interior whereas microwave absorption is sensitive to the surface of the grains, mostly influenced by interphase reactions.

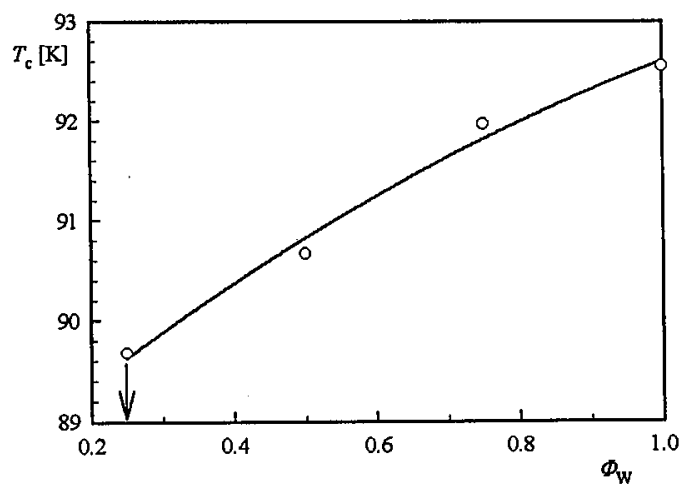

Fig. 5. The critical temperature $T_{\mathrm{c}}$ dependence on the YBCO nominal weight ratio $\Phi_{\mathrm{W}}$.

The critical temperature decreases by $3 \mathrm{~K}$ in the YBCO content range: $\Phi_{\mathrm{W}}=$ $1.0 \div 0.25$. The sample $\Phi_{\mathrm{W}}=0.2$ is nonsuperconducting.

The other authors confirm our result. For example, the minor effect of Sc substitution was reported by Zuo et al. [12], Ku et al. [14], and Dou et al. [15] who concluded that the critical temperature is almost unchanged up to $x=0.5 \div 0.6$ for the $\mathrm{Y}_{1-x} \mathrm{Sc}_{x} \mathrm{Ba}_{2} \mathrm{Cu}_{3} \mathrm{O}_{7-\delta}$ samples. On the other hand, Vassilev [16] reported that partial substitution of $\mathrm{Y}$ by another ions leads to quasilinear depression of $T_{\mathrm{c}}$.

\section{Conclusions}

The magnetic measurements performed in normal state revealed existence of the paramagnetic moment in YBCO-PST composites. The rapid increase in the paramagnetic moment for $\Phi_{\mathrm{W}}<0.5$ may be explained by the interphase reactions and formation of the minor phases.

The EPR investigations revealed the strong anisotropic signal for $\Phi_{\mathrm{W}}=1.0$ characterised by axial symmetry $\left(g_{\|}>g_{\perp}\right)$. This is the evidence for elongated octahedral or square co-ordination. For $\Phi_{\mathrm{W}}=0.2$, the measured $g$-factor equal 
to $g \approx 2.13$ is the average of $g_{\|}$and $g_{\perp}$. In this case the co-ordination is the superposition of the two orbital states $\left|x^{2}-y^{2}\right\rangle$ and $\left|3 z^{2}-r^{2}\right\rangle$ as discussed in [8].

The decay of the superconductivity is apparent from the measurements of the superconducting volume ratio $\Phi_{\mathrm{V}}$. The gradual decay of the superconductivity as the PST content increases takes place. For samples of low YBCO weight ratio $\Phi_{\mathrm{W}}<0.25$ no global (the whole sample) and local (separated grains) superconductivity was observed.

\section{Acknowledgments}

The authors acknowledge Dr. W. Sadowski from Gdansk University of Technology for useful discussions and Eng. H. Gierszal for technical assistance during the experiment. This work was supported by the grant No. 7 T08D 01715 of the Committee for Scientific Research.

\section{References}

[1] S. Regnier, J. Marfaing, Physica C 235-240, 1513 (1994).

[2] P. Lampin, PhD. thesis, Marseille 1994.

[3] Inorganic Phases, Powder Diffraction File, JCPDS, Swarthmore (Pa 19081, USA) 1992.

[4] B. Andrzejewski, J. Stankowski, A. Kaczmarek, B. Hilczer, J. Marfaing, in: Proc. Joint 29th AMPERE-13th ISMAR Int. Conf., Germany, Berlin 1998, p. 1224.

[5] U. Yaron, Y. Kornyushin, I. Felner, Phys. Rev. B 46, 14823 (1992).

[6] J. Marfaing, S. Regnier, J.M. Debierre, C. Caranoni, Physica C 280, 21 (1997).

[7] B. Andrzejewski, A. Kaczmarek, J. Stankowski, B. Hilczer, J. Marfaing, C. Caranoni, Physica C 284-288, 626 (2000).

[8] J. Stankowski, W. Kempiński, Z. Trybuła, Acta Phys. Pol. A 80, 571 (1991).

[9] S.K. Hoffmann, B. Czyżak, J. Stankowski, Acta Phys. Pol. A 77, 621 (1990).

[10] D. Berling, B. Loegel, A. Mehdaoui, S. Regnier, C. Caranoni, J. Marfaing, Supercond. Sci. Technol. 11, 1292 (1998).

[11] G. Svensson, Z. Hegedus, L. Wang, Ö. Rapp, Physica C 153-155, 864 (1988).

[12] F. Zuo, X.D. Chen, A. Chakraborty, B.R. Patton, J.R. Gaines, A.J. Epstain, Solid State Commun. 68, 2391 (1988).

[13] M. Krupski, J. Stankowski, S. Przybyl, B. Andrzejewski, A. Kaczmarek, B. Hilczer, J. Marfaing, C. Caranoni, Physica C 320, 120 (1999).

[14] H.C. Ku, J.R. Hwang, M.F. Tai, Physica C 153-155, 920 (1988).

[15] S.X. Dou, A.J. Bourdillon, X.Y. Sun, H.K. Liu, J.P. Zhou, N. Savvides, C.C. Sorrell, K.E. Easterling, Phys. Status Solidi B 147, K153 (1988).

[16] P.G. Vassilev, Physica C 153-155, 868 (1988). 